\section{Meliolaceae of Kerala, India - XXVIII}

\section{V.B. Hosagoudar \& G.R. Archana}

Tropical Botanic Garden and Research Institute, Palode - 695562 , Thiruvananthapuram, Kerala, India

Email: vbhosagoudar@rediffmail.com

\section{Meliola aristolochigena sp. nov}

(Fig. 1)

Coloniae epiphyllae, tenues vel densae, ad $2 \mathrm{~mm}$ diam Hyphae subrectae vel flexuosae, alternatim, opposite vel irregulariter acuteque vel laxe ramosae, laxe vel dense reticulatae, cellulae 12-16 x 8-10 $\mu \mathrm{m}$. Appressoria alternata vel ad 3\% opposita, antrorsa vel subantrorsa, 14-20 $\mu \mathrm{m}$ longa; cellulae basilares cylindraceae vel cuneatae, 3-7 $\mu$ m longae; cellulae apicales ovatae vel globosae, integrae, 11-13 x 9-13 $\mu \mathrm{m}$. Phialides appressoriis mixtus, alternatae vel oppositae, ampulliformes, 16-20 x 8-10 $\mu \mathrm{m}$. Setae myceliales dispersae, simplices, rectae, ad apicem acutae vel obtusae, ad 540 $4 \mathrm{~m}$ longae Perithecia dispersa, ad $120 \mu \mathrm{m}$ diam.; ascosporae oblongae vel cylindraceae, 4-septatae, constrictae ad septatae, 35-40 x 12$14 \mu \mathrm{m}$.

Colonies epiphyllous, thin to dense, up to $2 \mathrm{~mm}$ in diam. Hyphae substraight to flexuous, branching alternate, opposite to irregular at acute to wide angles, loosely to closely reticulate, cells 12-16 x 8-10 $\mu \mathrm{m}$. Appressoria alternate to about $3 \%$ opposite, antrorse to subantrorse, 14-20 $\mathrm{m}$ long; stalk cells cylindrical to cuneate, $3-7 \mu \mathrm{m}$ long; head cells ovate to globose, entire, 11-13 x 9-13 $\mu \mathrm{m}$. Phialides mixed with appressoria, alternate to opposite, ampulliform, 16-20 x 8-10 $\mu \mathrm{m}$. Mycelial setae scattered, simple, straight, acute to obtuse at the tip, up to $540 \mu \mathrm{m}$ long. Perithecia scattered, up to $120 \mu \mathrm{m}$ in diam.; ascospores oblong to cylindrical, 4-septate, constricted at the septa, 35-40 x 12-14 $\mu \mathrm{m}$.

Date of online publication 26 June 2009

ISSN 0974-7907 (online) | 0974-7893 (print)

Editor: Richard K. Mibey

\section{Manuscript details:}

Ms \# 02057

Received 13 September 2008

Final version received 07 April 2009

Finally accepted 20 May 2009

Citation: V.B. Hosagoudar \& G.R. Archana (2009). Meliolaceae of Kerala, India - XXVIII. Journal of Threatened Taxa 1(6): 348-350.

Copyright: (C) V.B. Hosagoudar \& G.R. Archana 2009. Creative Commons Attribution 3.0 Unported License. JoTT allows unrestricted use of this article in any medium for non-profit purposes, reproduction and distribution by providing adequate credit to the authors and the source of publication.

Acknowledgements: We thank Director, TBGRI, Palode for the facilities and Drs. M. Abdul Jabbar and E.S. Santhosh Kumar, TBGRI, Palode for their help in identifying the host plants. Dr. B. Shivaraju, Chief Conservator of Forests, Govt. of Kerala is greatly acknowledged for the facilities during the collection trip to Silent Valley National Park. We are grateful to Ministry of Environment and Forest, New Delhi for the financial support and to Forest Department, Govt. of Kerala for the forest permission.

\section{OPEN AGGESS | FREE DOWNLOAD}

\section{Material examined}

Type: 13.xii.2003, On leaves of Aristolochia tagala Cham. (Aristolochiaceae), Sairandhri, Silent Valley, Palghat, Kerala, India, coll. V.B. Hosagoudar et al. HCIO 46237 (type), TBGT 1649 (isotype), (MycoBank \# MB 513494).

Having opposite appressoria, Meliola aristolochigena can be compared with $M$. catharinensis Hansf. reported on Aristolochia triangularis from Brazil (Hansford 1961). However, differs from it in having distinctly longer appressoria (14-20 $\mu \mathrm{m}$ against $11-15 \mu \mathrm{m})$ and mycelial setae $(540 \mu \mathrm{m}$ against $230 \mu \mathrm{m})$

\section{Meliola pycnosporae sp. nov.}

(Fig. 2)

Coloniae amphigenae, caulicolae, densae, ad $2 \mathrm{~mm}$ diam. Hyphae subrectae, flexuosae vel anfractuae, alternatim, opposite vel irregulariter acuteque vel laxe ramosae, laxe vel dense reticulatae, cellulae 19-28 x 6-8 $\mu \mathrm{m}$. Appressoria alternata, ad $2 \%$ opposita, antrorsa vel subantrorsa, $11-16 \mu \mathrm{m}$ longa; cellulae basilares cylindraceae vel cuneatae, $3-5 \mu \mathrm{m}$ longae; cellulae apicales ovatae, globosae, rectae vel curvulae, integrae, 8-11 x 7-10 $\mu \mathrm{m}$. Phialides appressoriis mixtus, alternatae vel oppositae, ampulliformes, 19-24 x 8-10 $\mu \mathrm{m}$. Setae myceliales paucae, dispersae, simplices, rectae, sursum obtusae, ad $300 \mu \mathrm{m}$ longae. Perithecia dispersa, ad $120 \mu \mathrm{m}$ diam.; ascosporae oblongae, cylindraceae, 4-septatae, ad septatae leniter constrictae, 30-32 x 9-11 $\mu \mathrm{m}$.

Colonies amphigenous, caulicolous, dense, up to $2 \mathrm{~mm}$ in diam. Hyphae substraight, flexuous to crooked, branching alternate, opposite to irregular at acute to wide angles, loosely to closely reticulate, cells 19-28 x 6-8 $\mu \mathrm{m}$. Appressoria alternate,

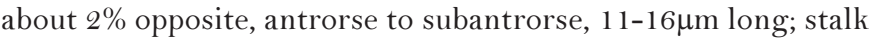
cells cylindrical to cuneate, $3-5 \mu \mathrm{m}$ long; head cells ovate,

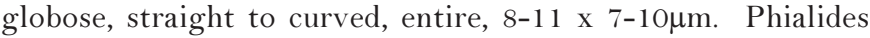
mixed with appressoria, alternate to opposite, ampulliform, 19-24 x 8-10 $\mu \mathrm{m}$. Mycelial setae few, scattered, simple, straight, obtuse at the tip, up to $300 \mu \mathrm{m}$ long. Perithecia scattered, up to $120 \mu \mathrm{m}$ in diam.; ascospores oblong, cylindrical, 4-septate, very slightly constricted at the septa, 30-32 x 9-11 $\mu \mathrm{m}$.

\section{Material examined}

Type: 14.xii.2003, On leaves of Pycnospora lutescens (Poir.) Schindl. (Fabaceae), on the way to Chempathy, Silent Valley, Palghat, Kerala, coll. V.B. Hosagoudar et al. HCIO 46244 (type), TBGT 1656 (isotype), (MycoBank \# MB 513495).

Based on the Beeli formula 3113.3221 , this species is close to Meliola erythrinae-microptericis Hansf. known on Erythrina micropteryx from San Domingo, but differs from it in having only $2 \%$ opposite appressoria, have straight setae and distinctly narrow ascospores (Hansford 1961).

\section{Meliola sairandhriana sp. nov.}

(Fig.- 3)

Coloniae amphigenae, plerumque hypophyllae, densae, velutinae, ad $5 \mathrm{~mm}$ diam. Hyphae rectae vel subrectae, alternatim vel unilateralis acuteque vel laxe ramosae, laxe vel arcte reticulatae, cellulae 19-32 x 6-8 $\mu \mathrm{m}$. Appressoria opposita, 


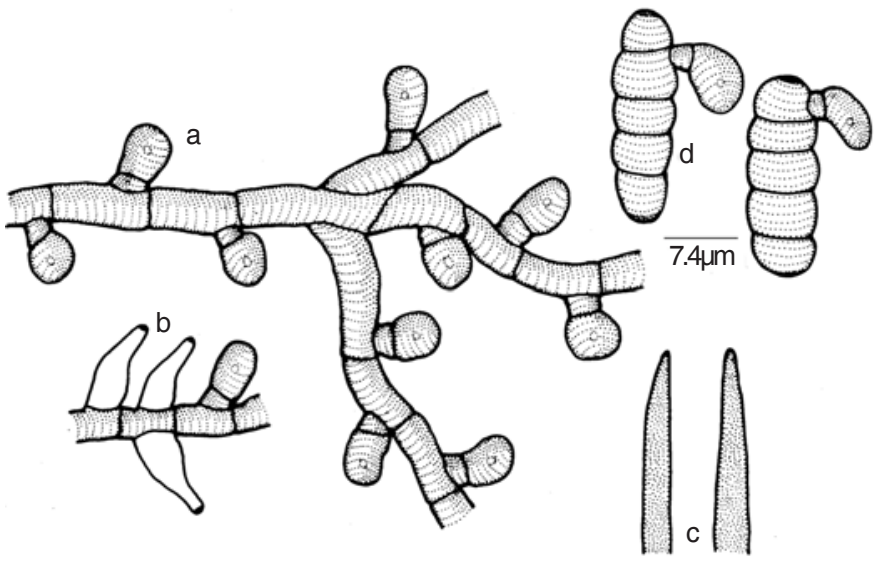

Figure 1. Meliola aristolochigena sp. nov.

a - Appressorium; b - Phialide; c - Apical portion of the mycelial setae; d - Ascospores

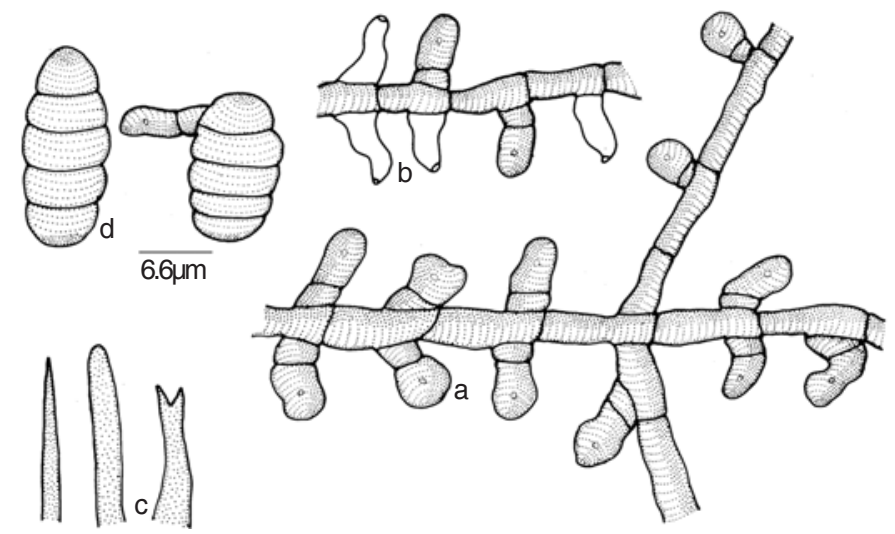

Figure 3. Meliola sairandhriana sp. nov.

a - Appressorium; b - Phialide; c - Apical portion of the mycelial setae; d - Ascospores

raro solitaria, recta vel curvula, antrorsa, subantrorsa vel retrorsa, 14-18 $\mathrm{m}$ longa; cellulae basilares cylindraceae vel cuneatae, 3-5 $\mu \mathrm{m}$ longa; cellulae apicales ovatae, oblongae, cylindraceae, integrae, angularis, sublobatae vel saepe bilobatae, 11-13 x 8-12 $\mu \mathrm{m}$. Phialides appressoriis mixtus, alternatae vel oppositae, ampulliformes, 19-23 x 8-10 $\mu \mathrm{m}$. Setae myceliales numerosae, circa peritheciis aggregatae, simplices, sigmoideae, ad sursum curvulae vel uncinatae, ad apicem acutae, obtusae vel bifidae, ad $345 \mu \mathrm{m}$ longae. Perithecia laxe aggregata, ad $196 \mu \mathrm{m}$ diam.; ascosporae ovatae, 4-septatae, constrictae ad septatae, 36-40 x 20-23 $\mu \mathrm{m}$.

Colonies amphigenous, mostly hypophyllous, dense, velvety, up to $5 \mathrm{~mm}$ in diam. Hyphae straight to substraight, branching alternate to unilateral at acute to wide angles, loosely to closely reticulate, cells 19-32 x 6-8 $\mu \mathrm{m}$. Appressoria opposite, rarely solitary, straight to curved, antrorse, subantrorse to retrorse, 14-18 $\mu \mathrm{m}$ long; stalk cells cylindrical to cuneate, 3-5 $\mu \mathrm{m}$ long; head cells ovate, oblong, cylindrical, entire, angular, sublobate to often bilobed, 11-13 x 8-12 $\mu \mathrm{m}$. Phialides mixed with appressoria, alternate to opposite, ampulliform, 19-23 x 8-10 $\mu \mathrm{m}$. Mycelial setae many, grouped around perithecia, simple, sigmoid, curved to uncinate at the apical portion, acute, obtuse to bifid at the tip, up to $345 \mu \mathrm{m}$ long. Perithecia loosely grouped, up to $196 \mu \mathrm{m}$ in diam.; ascospores oval, 4-septate, constricted at

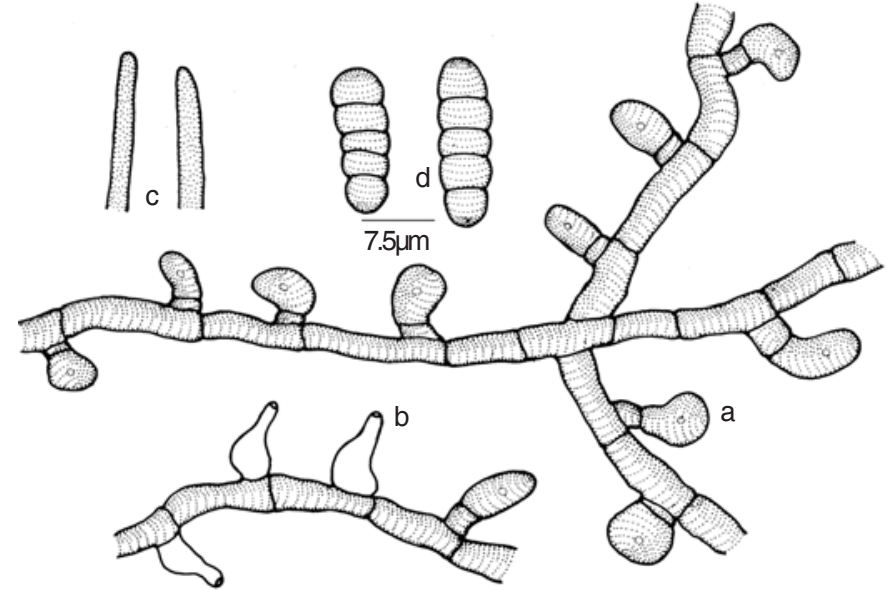

Figure 2. Meliola pycnosporae sp. nov.

a - Appressorium; $b$ - Phialide; $c$ - Apical portion of the mycelial setae; d - Ascospores

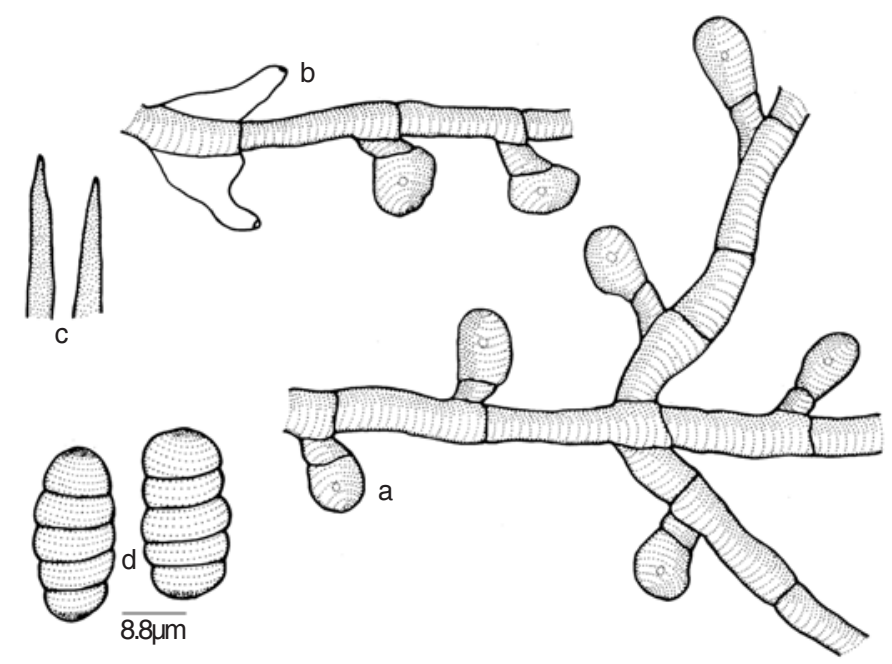

Figure 4. Meliola strebli sp. nov.

a - Appressorium; b - Phialide; c - Apical portion of the mycelial setae; d - Ascospores

the septa, 36-40 x $20-23 \mu \mathrm{m}$.

\section{Material examined}

Type: 13.xii.2003, On leaves of Aglaia minutiflora Bedd. (Meliaceae), Sairandhri, Silent Valley, Palghat, Kerala, India, coll. V.B. Hosagoudar et al. HCIO 46139 (type), TBGT 1902 (isotype), (MycoBank \# MB 513496).

Based on the uncinate mycelial setae, M. sairandhriana can be compared to $M$. reinwardtiodendri Hosag. known on Reinwardtiodendron anamallayanam from the Western Ghats region but differs from it in having amphigenous colonies, simple to dentate mycelial setae and smaller ascospores (Hosagoudar 1996).

\section{Meliola strebli sp. nov. \\ (Fig.-4)}

Coloniae amphigenae, plerumque hypophyllae, densae, velutinae, ad $3 \mathrm{~mm}$ diam. confluentes. Hyphae rectae vel 
subrectae, alternate vel opposite acuteque vel laxe ramosae, laxe vel dense reticulatae, cellulae 20-24 x 6-8 $\mu \mathrm{m}$. Appressoria alternata vel unilateralis, sparse posita, antrorsa, subantrorsa vel raro retrorsa, $17-21 \mu \mathrm{m}$ longa; cellulae basilares cylindraceae vel cuneatae, 4-6 $\mu \mathrm{m}$ longae; cellulae apicales ovatae, globosae, integrae, rectae vel curvulae, 11-15 x 9-11 $\mu \mathrm{m}$. Phialides appressoriis mixtus, alternatae vel oppositae, ampulliformes, 16-19 x 8-10 $\mu \mathrm{m}$. Setae myceliales dispersae, simplices, rectae, acutae ad apicem, ad $735 \mu \mathrm{m}$ longae. Perithecia dispersa vel laxe aggregata, ad $212 \mu \mathrm{m}$ diam.; ascosporae oblongae, 4-septatae, leniter constrictae ad septatae, 40-44 x 16-18 $\mu \mathrm{m}$.

Colonies amphigenous, mostly hypophyllous, dense, velvety, up to $3 \mathrm{~mm}$ diam., confluent. Hyphae straight to substraight, branching alternate to opposite at acute to wide angles, loosely to closely reticulate, cells 20-24 x 6-8 $\mu \mathrm{m}$. Appressoria alternate to unilateral, distantly placed, antrorse, subantrorse to rarely retrorse, 17-21 $\mu \mathrm{m}$ long; stalk cells cylindrical to cuneate, 4$6 \mu \mathrm{m}$ long; head cells ovate, globose, entire, straight to curved, 11-15 x 9-11 $\mu \mathrm{m}$. Phialides mixed with appressoria, alternate to opposite, ampulliform, 16-19 x 8-10 $\mu \mathrm{m}$. Mycelial setae scattered, simple, straight, acute at the tip, up to $735 \mu \mathrm{m}$ long. Perithecia scattered to loosely grouped, up to $212 \mu \mathrm{m}$ diam.; ascospores oblong, 4-septate, slightly constricted at the septa, $40-44 \times 16-18 \mu \mathrm{m}$

\section{Material examined}

Type: 23.xi.2004, On leaves of Streblus taxoides (Heyne ex
Roth) Kurz (Moraceae), near Sasthanada tribal colony, Sankili forest, Shendhurney Wildlife Sanctuary, Kollam, Kerala, India, coll. V.B. Hosagoudar et al. HCIO 46162 (type), TBGT 1574 (isotype); TBGT 1605, HCIO 46193; 23.xi.2004, S. asper Lour., Sankili forest, Shendhurney Wildlife Sanctuary, Kollam, Kerala, coll. V.B. Hosagoudar et al. TBGT 1889, HCIO 46126; 23.xi.2004, Streblus sp., Tribal colony, Sasthanamada, Sankili forest, Shendhurney Wildlife Sanctuary, Kollam, coll. V.B. Hosagoudar et al. TBGT 1893, HCIO 46130, (MycoBank \# MB 5 13497).

Based on the digital formula 3111.4233 , this species can be compared with Meliola ficium Yates and its variety var. ugandensis Hansf. However, M. strebli differs from $M$. ficium in having narrow ascospores and having longer mycelial setae (3111.4333). It also differs from M. ficium Yates var. ugandensis Hansf. in having shorter appressoria and larger ascospores (3111.4223) (Hansford 1961).

\section{References}

Hansford, C.G. (1961). The Meliolineae. A Monograph. Sydowia. Beih. 2: $1-806$

Hosagoudar, V.B. (1996). Meliolales of India. Botanical Survey of India, Calcutta, 363pp.

Hosagoudar, V.B. \& H. Biju (2006). Studies on foliicolous fungi - XXII Microfungi of Silent Valley National Park, Palghat district in Kerala State. Journal of Mycopathol. Research 44: 39-48. 